\title{
Huella de Carbono de Organización y Producto con Enfoque Híbrido: Mejoras en el Método Compuesto de las Cuentas Contables
}

\author{
Alvarez S. * \\ ETSI Montes, Forestal y del Medio Natural. Depto. Silvopascicultura. Universidad Politécnica de Madrid. \\ *e-mail: Sergio.alvarez@upm.es
}

La lucha contra el cambio climático supone uno de los retos ambientales más importantes de este siglo XXI. Ante esta situación, los esfuerzos se han desarrollado situando al productor, y no al consumidor, como responsable del problema. Estas estrategias son claramente inefectivas dando lugar al fenómeno conocido como fuga de carbono que tiene como consecuencia el aumento de las emisiones de gases de efecto invernadero en países con escasa o nula legislación ambiental. El concepto de la Huella de Carbono nace con la finalidad de situar la responsabilidad en el consumidor final de bienes y servicios, independientemente del lugar donde se desarrolle la producción. De esta manera se pretende desarrollar una herramienta válida para que empresas y consumidores funcionen como motores de cambio hacia una economía dinamizada por la búsqueda del desarrollo sostenible.

Para alcanzar el objetivo de reducir las emisiones de gases de efecto invernadero es necesario que la Huella de Carbono pueda ser aplicable a todas las actividades de la economía. Sin embargo, una de las principales dificultades halladas al respecto es la diferencia de enfoques para el cálculo de la Huella de Carbono de producto y la Huella de Carbono de organización. De igual manera existen importantes dificultades en el establecimiento de los límites del sistema en estudio. Para asegurar el éxito de la implantación de la Huella de Carbono en la sociedad, es necesario el establecimiento de los mismos criterios en los distintos estudios. De lo contrario, la comparación no será posible y, por lo tanto, la confianza del consumidor se verá comprometida.

Los últimos avances relevantes en el cálculo de la Huella de Carbono se basan en dos propuestas diferentes: El Análisis de Ciclo de Vida y la Extensión Ambiental del Análisis Input-Output. Ambas metodologías tienen importantes aspectos positivos y negativos. Por lo tanto, la hibridación entre ambos enfoques supone una clara oportunidad en la búsqueda de sinergias. En respuesta a esta demanda, diferentes herramientas de enfoque híbrido están siendo concebidas. La investigación lle- 
vada a cabo en esta tesis doctoral parte del avance desarrollado en la concepción del Método Compuesto de las Cuentas Contables (MC3). El MC3 es un método de análisis híbrido por niveles que desarrolla un cálculo de la Huella de Carbono de organización para el posterior cálculo de la Huella de Carbono parcial de producto.

Esta investigación tiene como objetivo general evaluar el MC3 como herramienta de cálculo de la Huella de Carbono, válida tanto para organización como para producto. Para ello se analizan pormenorizadamente cuatro casos de estudios con características innovadoras.

El primer caso de estudio determina la huella de carbono de la Escuela Técnica Superior de Ingenieros de Montes (Universidad Politécnica de Madrid). Según la aplicación del MC3, la huella de carbono total de esta Escuela en 2010 fue de 2147 t $\mathrm{CO}_{2}$ eq, de los cuales $59.0 \%$ corresponde a las denominadas emisiones de alcance 3 , emisiones de voluntaria cuantificación. Debido a la amplia gama de categorías de consumo consideradas, el MC3 permite capturar posibles cambios en los patrones de consumo. La cuantificación de la Huella de Carbono proporciona una base analítica para la ejecución de planes de gestión del carbono en el centro universitario. Este trabajo ha dado lugar a la integración de los nuevos esquemas de desarrollo sostenible en la ETSIM.

El segundo caso de estudio analiza las diferencias metodológicas entre el enfoque de la Huella de Carbono de producto y el enfoque de la Huella de Carbono corporativa. Esta divergencia metodológica dificulta seriamente la implantación del indicador. El MC3 permite ser aplicado con el mismo esquema de cálculo a organizaciones y productos. Para analizar la viabilidad y fortalezas del MC3, se compara su aplicación con el desarrollo de un Análisis de Procesos, herramienta clásica del Análisis de Ciclo de Vida. Para desarrollar el estudio se emplea un productor español de pallets de madera que produce y comercializa 1.6 millones de unidades. La implementación de ambos métodos revela que: (1) las emisiones del MC3 son 22\% más altas que las derivadas del Análisis de Procesos, (2) el Análisis de Procesos permite el desglose con mayor detalle en las fases del ciclo de vida y (3) el tiempo de cálculo se reduce drásticamente con el MC3. Por lo tanto, el MC3 puede mejorar los actuales trabajos realizados tanto en los dos enfoques (producto y organización), estimulando la innovación y el apoyo al consumo sostenibles.

El tercer caso de estudio se centra en un escenario internacional analizando la Huella de Carbono de un parque de energía eólica en Brasil. La aplicación del MC3 arroja unas emisiones de 18,33 gr $\mathrm{CO}_{2}$ eq por $\mathrm{kWh}$ entregado a la red eléctrica nacional de Brasil. Este resultado difiere de los anteriormente publicados debido a que la aplicación del MC3 ha permitido tener en consideración fases y áreas del ciclo de vida nunca antes descritas, como por ejemplo la promoción del parque y la construcción de accesos y drenajes. En este estudio, el valor comparable derivado de la fabricación, el transporte y la construcción de los aerogeneradores se reduce a menos de la mitad, 8,42 gr $\mathrm{CO}_{2}$ eq por kWh. De acuerdo con los resultados, el MC3 es un método práctico que permite la definición de un objetivo más amplio y alcance. Su aplicación al caso de estudio demuestra la idoneidad del método para su implementación en grandes proyectos internacionales. 
El cuarto caso de estudio plantea cómo el comercio internacional ha jugado históricamente un papel importante en el desarrollo económico. Sin embargo, la falta del pleno y uniforme análisis de las externalidades ambientales negativas, hace que la producción pueda tener lugar en regiones con escasa o nula legislación ambiental. Esta fuga de carbono puede presentar al comercio internacional como un gran impulsor de las emisiones de gases de efecto invernadero. El objetivo de este trabajo es el análisis del comercio internacional como un impulsor de las emisiones de $\mathrm{CO}_{2}$. La influencia se evalúa en función de los territorios y sectores económicos. Dos escenarios (de referencia y economías cerradas) se construyen para evaluar el hipotético cierre al comercio internacional de bienes y servicios, obligando a cada país a producir nacionalmente lo que consume como demanda final. Los resultados en el escenario cerrado muestran una ligera disminución de las emisiones, tan solo 18 Mt CO2eq. Sin embargo, esta ligera diferencia esconde importantes intercambios de contaminación. Los resultados se desglosan por nivel económico nacional, las emisiones asignables a los países Anexo 1 se reducirían en $434 \mathrm{Mt} \mathrm{CO}_{2}$ eq y las emisiones en países no-Anexo 1 aumentarían en $416 \mathrm{Mt} \mathrm{CO2eq.} \mathrm{Según} \mathrm{este} \mathrm{análisis,} \mathrm{una}$ tasa verde a la importación ayudaría a los países desarrollados como Estados Unidos y Japón a reducir sus emisiones de GEI en más de $110 \mathrm{Mt} \mathrm{CO}_{2}$ eq cada uno. Según el análisis de sectores económicos, se lograría una reducción de $209 \mathrm{Mt}$ $\mathrm{CO}_{2}$ eq mediante el cierre de la electricidad en cada país del Anexo 1. Estos resultados tienen importantes implicaciones políticas. Sin embargo, es importante considerar que el cierre de las economías puede tener interacciones significativas. Este estudio refuerza aún más la necesidad de elaborar de manera oficial inventarios basados en el consumo.

Estos estudios son discutidos en su conjunto a fin de poner en valor las fortalezas de las innovaciones implementadas con un sentido integrador. También se proponen estrategias futuras que permitan mejorar la propuesta metodológica del MC3 con el punto de mira puesto en la internacionalización y la armonización con los estándares internacionales de la Huella de Carbono. Según la experiencia desarrollada, el MC3 es un método de cálculo de la Huella de Carbono práctico y válido para evaluar la cantidad de emisiones directas e indirectas de gases de efecto invernadero de cualquier tipo de actividad. Una de las principales conclusiones es que el MC3 puede ser considerado una herramienta válida para el ecoetiquetado global de bienes y servicios que permita, tanto a empresas como a consumidores, funcionar como motores de cambios hacia una economía dinamizada por la búsqueda de la racionalización de recursos. 


\section{Datos de la tesis}

Accésit SECF a la mejor Tesis Doctoral 2014.

Director: Agustín Rubio (Universidad Politécnica de Madrid).

Centro: Universidad Politécnica de Madrid.

Fecha de defensa: Junio de 2014

\section{Publicaciones derivadas de la tesis doctoral}

Alvarez, S., Blanquer, M., Rubio, A., 2014. Carbon footprint using the Compound Method based on Financial Accounts. The case of the School of Forestry Engineering, Technical University of Madrid. J. Clean. Prod. 66, 224-232. doi:10.1016/ j.jclepro.2013.11.050

Alvarez, S., Carballo-Penela, A., Mateo-Mantecon, I., Rubio, A., 2015. Unique Approach for Carbon Footprint Analysis: Recommendations and Guidelines Based on the Integrated Approach. J. Clean. Prod. under revi.

Alvarez, S., Planelles, R., Rubio, A., 2015a. Carbon footprint from helitankers. Sustainable decision-making in aerial wildfire fighting. Int. J. Wildl. Fire accepted.

Alvarez, S., Rubio, A., 2015a. Carbon Footprint in Green Public Procurement: A case study in the services sector. J. Clean. Prod. 93, 159-166. doi:10.1016/j.jclepro.2015.01.048

Alvarez, S., Rubio, A., 2015b. Compound Method Based on Financial Accounts versus Process Life Cycle Analysis in Product Carbon Footprint: a Comparison Using Wood Pallets. Ecol. Indic. 49, 88-94. doi:10.1016/j.ecolind.2014.10.005

Alvarez, S., Rubio, A., Barrett, J., Owen, A., 2012. Isolating economies. Is trade a large CO2 driver?, in: The 20th International Input- Output Conference. Bratislava (Slovakia).

Alvarez, S., Sosa, M., Rubio, A., 2015b. Product and corporate carbon footprint using the compound method based on financial accounts. The case of Osorio wind farms. Appl. Energy 139, 196-204. doi:10.1016/j.apenergy.2014.11.039. 